\title{
Rainfall-Runoff Model(s) Performance in the Blue Nile River
}

\author{
Bashar K.E. ${ }^{1}$, PhD.
}

\section{Abstract}

This paper presents the performance of some tested rainfall-runoff models to forecast river flows of the Blue Nile catchment up to Eddeim.

Flow forecasting is an important step in river basin management in particular and water resources management in general. River flow models are used as components in actual flow forecasting schemes. They are also used in providing for efficient operation of storage reservoirs. Usually, flow forecasts are obtained in real time by transforming the input into a discharge using models. These forecasts may subsequently be modified or updated in accordance with the errors observed in the previous forecasts up to the time of making the new forecast.

The system analysis or black box approach depends on a prior assumption of flexible linear and time invariant relationship the expression of which can be obtained by the application of systems analysis approach to records. The conceptual model provides an alternative approach in which the input-output transformation goes through a series of steps.

In this study, systems and conceptual modelling techniques are applied to the Blue Nile catchment up to Eddeim of the Ethiopian high lands. The models were applied in non-parametric and parametric forms. Parameter optimisation is carried out by ordinary least squares, Rosenbrock, Simplex and genetic algorithm.

The areal rainfall which is the main input to these models was estimated using arithmetic mean. However, attempts to estimate the areal rainfall by the Thiesen polygon method was made but the improvement in the model performance can not justify the amount of work involved in making Thiesen's estimate.

It is shown that the simple assumption of linearity is not adequate in modelling the rainfall runoff transformation. However, in the Blue Nile catchment, which exhibits a marked seasonal behaviour, good results were obtained with Linear Perturbation Model (LPM) which involves the assumption of linearity between the departures from seasonal expectations in input and output series.

The application of the GFFS (collection of systems and conceptual models) software proved to be possible with variable efficiencies. The LPM in non-parametric or parametric form, the LVGF model, the ANN and the SMAR model can be used to forecast (reproduce) Ed-deim flows with high performance.

Within the range of the tested models LPM was found to be the best candidate model that can forecast the flows under a wide range of conditions of marked seasonality by accounting for more than $90 \%$ of the initial variance.

\footnotetext{
${ }^{1}$ UNESCO Chair in Water Resources, Omdurman Islamic University.
} 


\section{المستخلص}

هذا البهث بهلي ظلرة عالمة عن إلستخدلم النزجة الهيرولكية للتنباً بالجريلن في حوض نهر النل

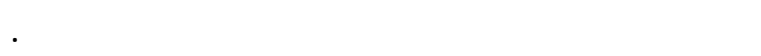

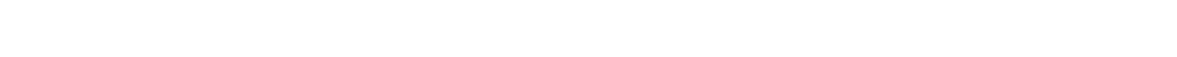

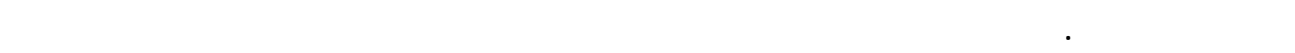

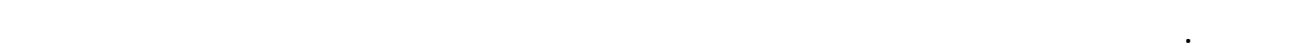

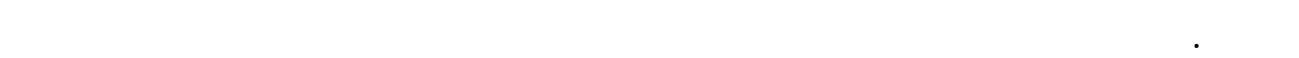
موعد التنبأ الجنيد.

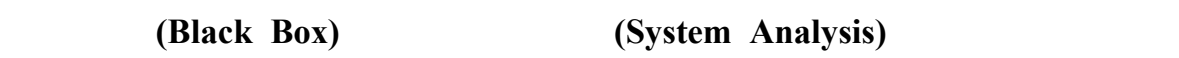

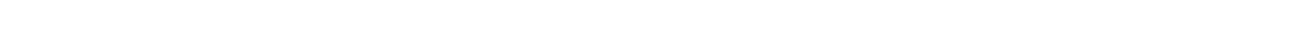

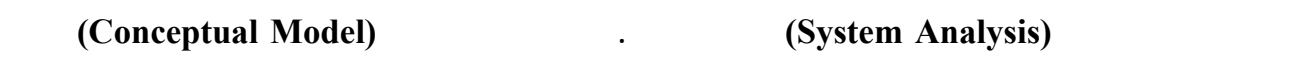

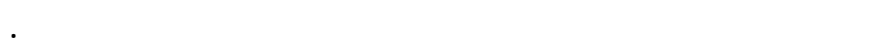

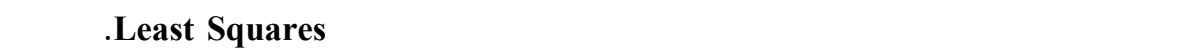

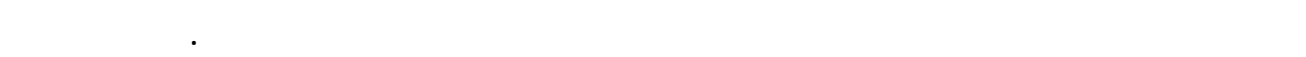

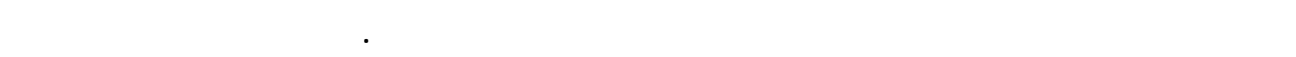

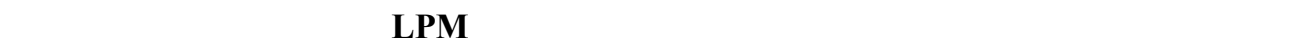
خلية بين بول الفولرق الموتمية لإمخلات والمخرجلت.

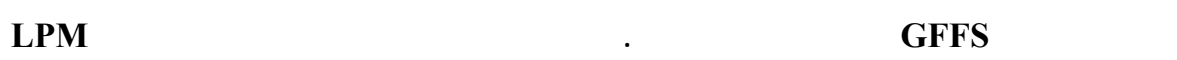

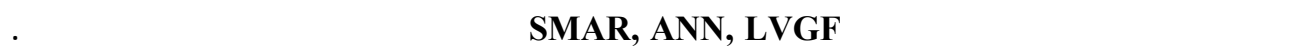

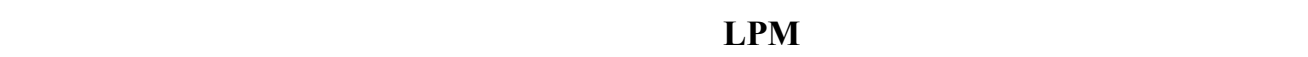

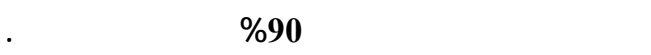

\section{Introduction}

In recent decades, the advent of increasingly efficient computing technology has provided hydrologists with exciting new tools for the mathematical modelling of hydrological systems including, but extending far beyond, the more traditional river-flow forecasting applications. Elaborate physically-based distributed modelling, and elegant mathematical techniques using Artificial Neural Networks, Fuzzy systems, Wavelets, etc. are being used, all with high levels of complexity, but not necessarily with increased levels of efficiency attainment, particularly in the context of flow forecasting. Most such exercises are certainly significant from a research 
point of view, as they attempt to throw more light on the physical processes involved, but data demands, lack of parsimony in model parameters, and structural complexity can still be a major deterrent when it comes to applying these models in real-life problem solving. In the flow-forecasting context, even simple black-box type system-theoretic models, or physically inspired lumped conceptual models, can produce better and more reliable flow forecasting results than complex distributed models.

The Galway River Flow Forecasting System (GFFS) is a software package developed at the Department of Engineering Hydrology, National University of Ireland, Galway [O'Connor et al, 2001]. It comprises a suite of models for simulation, updating and real-time forecasting applications. The degree of structural complexity, associated parameter parsimony, and difficulty in objective function evaluation of these models, varies considerably. The models and techniques used in the present study, all from the GFFS package, are applied to the Blue Nile catchment representing variability in geographic location, climatic conditions, areal extent and various physiographical characteristics. The Nash-Sutcliffe model efficiency criterion is used to judge the models performance.

The objective of the study is to carry out performance test of rainfallrunoff models suite in the GFFS software as applied to the Blue Nile River.

\section{The Candidate Models}

Three system-theoretic black-box models, an Artificial Neural Network Model, a simple conceptual Soil Moisture Accounting \& Routing Model and method of combination of outputs were used. Brief descriptions of these models are provided in the following section.

\subsection{The Simple Linear Model (SLM)}

\subsubsection{Non-parametric modelling}

The hypothesis of the SLM is introduced by Nash and Foley [1982]. The SLM approach assumes a linear time-invariant relationship between the total rainfall $R_{t}$ and the total discharge $Q_{t}$. The input-output relationship for lumped, linear, time invariant system expressed in terms of a series of pulses or mean values over successive short intervals $\mathrm{T}$, can be conveniently obtained from the response to unit pulse of duration $\mathrm{T}$ which is a convenient expression of the operation of the of the system. The discrete linear input-output relationship is expressed in terms of sampled pulse 
response by the convolution summation relation [Kachroo and Liang, 1992], which can be written after incorporating the model error term as

$$
Q_{t}=\sum_{j=1}^{m} R_{t-j+1} h_{j}+e_{t}
$$

where $Q_{t}$ and $R_{t}$ are the discharge and rainfall respectively at the $t^{\text {th }}$ timestep, $h_{j}$ is the $j^{\text {th }}$ discrete pulse response ordinate or weight, $m$ is the memory length of the system, and $e_{t}$ is the forecast error term.

\subsubsection{Parametric Modelling-the Gamma Function Model}

Constraint to the shape and volume of the estimated pulse response functions is obtained by parametric modelling where a solution is sought within the constraint of an assumed model form. Based on prior knowledge of the system behaviour the response function is represented by a suitable mathematical equation involving only a few parameters. The parameters must be estimated by optimization through a search in the space of reasonable parameter values.

The most popular impulse response function is given by

$$
h(t)=\frac{1}{k \Gamma(n)} e^{-\frac{t}{k}}\left(\frac{t}{k}\right)^{n-1}
$$

where $\Gamma(n)=\int_{0}^{\infty} e^{-x} x^{n-1} d x$ is the gamma function of $\mathrm{n}$.

The equation of the SLM for single input-single output will be

$$
Q_{t}=G_{f} \sum_{j=1}^{m} R_{t-j+1} h_{j}+e_{t}
$$

where $h_{j}$ is given by equation (2) and $G_{f}$ is the gain factor.

For multiple input-single output system under the constraints of the gamma function impulse response the parameters $n, n k$ and $G_{f}$ must be found for each input. 


\subsection{The Linear Perturbation Model (LPM)}

In the LPM [Nash and Barsi, 1983], it is assumed that, during a year in which the rainfall is identical to its seasonal expectation, the corresponding discharge hydrograph is also identical to its seasonal expectation. However, in all other years, when the rainfall and the discharge values depart from their respective seasonal expectations, these departures series are assumed to be related by a linear time invariant system. Thus the linear perturbation model uses the information contained in the observed seasonal variation of the hydrograph to reduce the dependence on linearity and increase the dependence on observed seasonal behaviour. The model assumes the following:

a. if each input function for each day of the year is equal to its expected value for that date, the output will also equal its expectation for that date

b. the perturbations or the departure from the date expected input values are linearly related to the corresponding perturbations or departures from the date expected output values.

For a single input, the relation between the departure (i.e. perturbation) series of the LPM has the convolution summation form and can be written as

$$
Q_{t}^{\prime}=\sum_{j=1}^{m} R_{t-j+1}^{\prime} h_{j}+e_{t}
$$

where $R_{t}^{\prime}=R_{t}-R_{d}$ and $Q_{t}^{\prime}=Q_{t}-Q_{d}$ are the respective departures of rainfall and discharge from their seasonal expectations, $e_{t}$ is the error output term and $\mathrm{d}=1,2,3, \ldots, 365$. Model-estimated departure values are added to the seasonal expectations to give the estimated discharge series.

\subsection{The Linearly Varying Gain Factor Model (LVGFM)}

The LVGFM, proposed by Ahsan and O'Connor [1994] for the single-input to single-output case, involves only the variation of the gain factor with the selected index of the prevailing catchment wetness, but not the shape (i.e. the weights) of the response function. Using a time-varying gain factor $G_{t}$, the model output has the structure 
$Q_{t}=G_{t} \sum_{j=1}^{m} R_{t-j+1} B_{j}+e_{t}$

where $\sum_{j=1}^{m} B_{j}=1$

In its simplest form, $G_{t}$ is linearly related to an index of the soil moisture state $z_{t}$ by the equation $G_{t}=a+b z_{t}$, where $a$ and $b$ are constants. The value of $z_{t}$ is obtained from the outputs of the naïve SLM, operating as an auxiliary model, using

$$
z_{t}=\frac{\hat{G}}{\bar{Q}} \sum_{j=1}^{m} R_{t-j+1} \hat{h}_{j}
$$

where $\hat{G}$ and $\hat{h}_{j}$ are estimates of the gain factor and the pulse response ordinates respectively of the SLM and $\bar{Q}$ is the mean calibration discharge.

\subsection{The Artificial Neural Network Model (ANNM)}

The "multi-layer feed-forward network" type of artificial neural network, used in this study, consists of an input layer, an output layer and only one "hidden" layer located between the input and the output layers [Shamseldin 1997]. Each neuron of a particular layer has connection pathways to all the neurons in the following adjacent layer, but none to those of its own layer or to those of the previous layer (if any).

Likewise, nodes in non-adjacent layers are unconnected. In the output layer, there is only one neuron, for the single output. Because the neural network itself does not incorporate storage effects, storage is implicitly accounted for by the use of the output series of the naïve SLM. For a neuron either in the hidden or in the output layer, each received input $y_{i}$ is transformed to its output $y_{o}$ by the mathematical transfer function:

$$
y_{o}=f\left(\sum_{i=1}^{M} w_{i} y_{i}+w_{o}\right)
$$

where $f()$ denotes the transfer function, $w_{i}$ are the input connection pathway weights, $M$ is the total number of inputs (which equals the number of 
neurons in the preceding layer), and $w_{o}$ is the neuron threshold (or bias). The non-linear transfer function adopted for the neurons of the hidden and output layers is the widely-used logistic/sigmoid function:

$$
f\left(\sum_{i=1}^{M} w_{i} y_{i}+w_{o}\right)=\frac{1}{1+e^{\sigma\left(\sum_{i=1}^{M} w_{i} y_{i}+w_{o}\right)}}
$$

bounded in the range $[0,1]$. The neuron weights $w_{i}$, the threshold $w_{o}$ and $\sigma$ can all be interpreted as parameters of the network configuration.

\subsection{The Soil Moisture Accounting And Routing (SMAR) Model}

The system type models such as SLM and LPM can fail to reproduce the observed hydrograph. The failure seems to lie in their model structure as no component that adequately accounts for evaporation and soil moisture effects in determining the volumes of runoff.

The SMAR Model is a development of the 'Layers' conceptual rainfall-runoff model introduced by O'Connell et al. [1970], its water balance component having been proposed in 1969 by Nash and Sutcliffe [Clarke 1994]. Using a number of empirical and assumed relations, which are considered to be at least physically plausible, the non-linear water balance (i.e. soil moisture accounting) component ensures satisfaction of the continuity equation, over each time-step. The routing component, on the other hand, simulates the attenuation and the diffusive effects of the catchment by routing the various generated runoff components through conservative linear time-invariant storage elements. For each time-step, the combined output of the two routing elements adopted (i.e. one for generated 'surface runoff' as input and the other for generated 'groundwater runoff' as input) becomes the simulated discharge forecast.

The water balance component of this model operates as a vertical stack of horizontal soil layers each can contain certain amount of water at field capacity see figure (1). Evaporation occurs from the top layer at a potential rate and from the second layer on exhaustion of the top layer at the remaining potential rate multiplied by a parameter $\mathrm{C}$ whose value is less than unity. On exhaustion of the second layer evaporation proceeds from the third layer at the remaining potential rate multiplied by $\mathrm{C}^{2}$ and so on. Thus, 
a constant potential evaporation rate applied to the basin reduces the soil moisture storage in a roughly exponential manner.

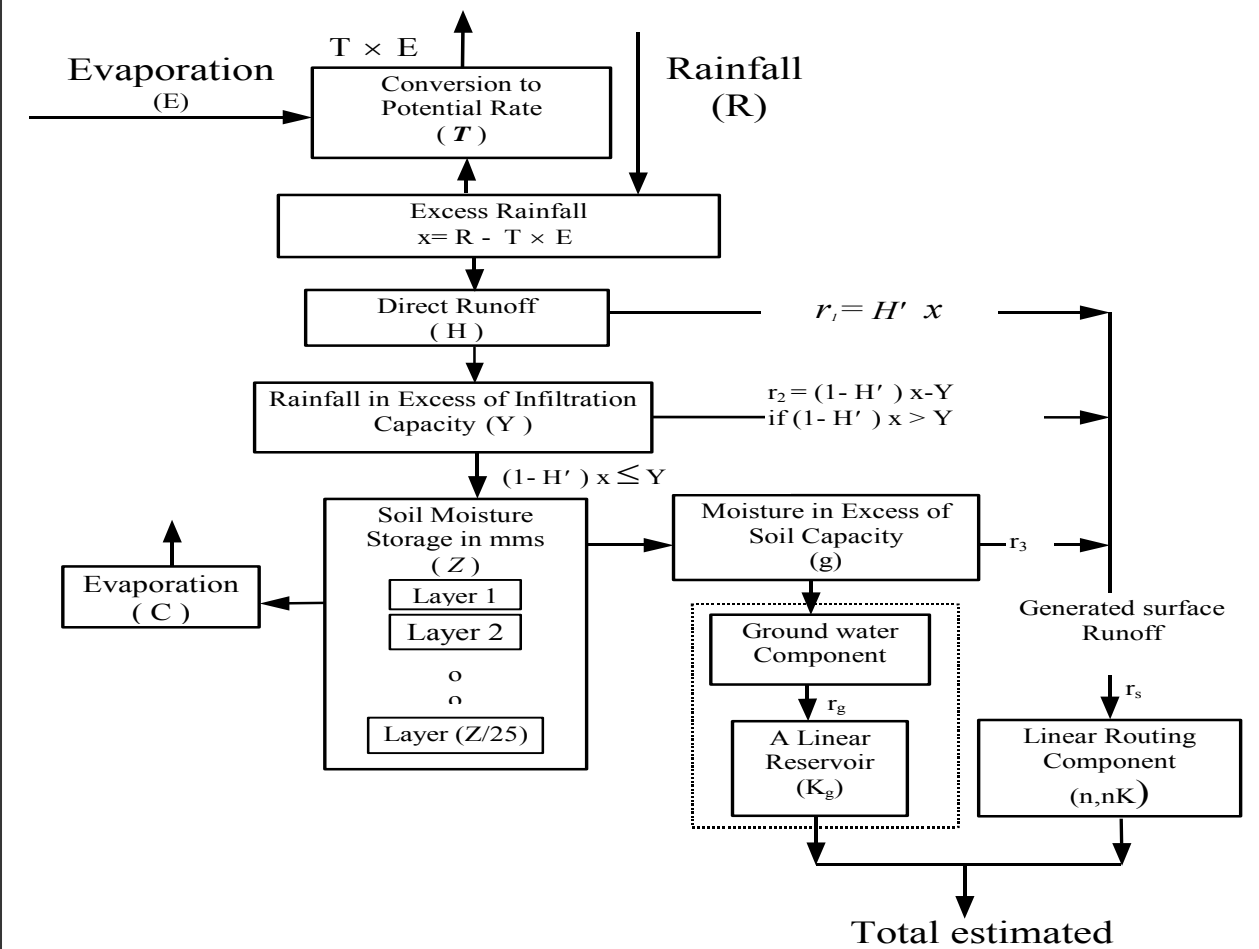

Figure (1): Schematic diagram of SMAR Model (Shamseldin et al 1997)

\subsection{Methods of Combining the Forecasts of Different Models}

Instead of relying on one individual model or switching between models, an alternative approach is to generate forecasts simultaneously from a number of different models and then combine these forecasts in an optimum manner. This can be done in several ways such as: 


\subsubsection{The Simple Average Method (SAM)}

This is the simplest method for combining the outputs of different individual models. Given the output of N models, a combined estimate of discharge $\hat{Q} c_{t}$ of the $\mathrm{t}^{\text {th }}$ time period using SAM is given by:

$$
\hat{Q} c_{t}=\frac{1}{N} \sum_{i=1}^{N} \hat{Q}_{i, t}
$$

SAM method given by equation (9) can produce forecasts that are better than those of the individual models (Shamseldin et al 1997, Makridakis et al 1982) and its accuracy depends mainly on the number of the models involved and on the actual forecasting ability of the specific models included in the simple average (Shamseldin et al 1997, Makridakis and Winkler 1983).

\subsubsection{The Weighted Average Method (WAM)}

The SAM method can be quite inefficient when some individual models selected for combination consistently produce more accurate forecasts than others (Shamseldin et al 1997, Armstrong 1989). In this case the use of a weighted average method would be preferable.

Granger and Ramanathan (1984) and Shamseldin et al (1997) used a weighted average method (WAM) for combining the outputs of $\mathrm{N}$ models using the formula:

$$
Q_{t}=\sum_{i=1}^{N} a_{i} \hat{Q}_{i, t}+e_{t}
$$

Where $Q_{t}$ is the observed discharge of the $t^{\text {th }}$ time period, $a_{i}$ is the weight assigned to the $\mathrm{i}^{\text {th }}$ model estimated discharge $\hat{Q}_{i, t}$ and $\mathrm{e}_{\mathrm{t}}$ is the combination error term.

Equation (10) can be expanded and written is a matrix form as: 


$$
\left[\begin{array}{c}
Q_{1} \\
Q_{2} \\
Q_{3} \\
\vdots \\
\vdots \\
Q_{k-1} \\
Q_{k}
\end{array}\right]=\left[\begin{array}{cccccc}
\hat{Q}_{1,1} & \hat{Q}_{2,1} & \cdots & \cdots & \hat{Q}_{N-1,1} & \hat{Q}_{N, 1} \\
\hat{Q}_{1,2} & \hat{Q}_{2,2} & \cdots & \cdots & \hat{Q}_{N-1,2} & \hat{Q}_{N, 2} \\
\hat{Q}_{1,3} & \hat{Q}_{2,3} & \cdots & \cdots & \hat{Q}_{N-1,3} & \hat{Q}_{N, 3} \\
\vdots & \vdots & \vdots & \vdots & \vdots & \vdots \\
\vdots & \vdots & \vdots & \vdots & \vdots & \vdots \\
\hat{Q}_{1, k-1} & \hat{Q}_{2, k-1} & \cdots & \cdots & \hat{Q}_{N-1, k-1} & \hat{Q}_{N, k-1} \\
\hat{Q}_{1, k} & \hat{Q}_{2, k} & \cdots & \cdots & \hat{Q}_{N-1, k} & \hat{Q}_{N, k}
\end{array}\right]\left[\begin{array}{c}
a_{1} \\
a_{2} \\
a_{3} \\
\vdots \\
\vdots \\
a_{N-1} \\
a_{N}
\end{array}\right]+\left[\begin{array}{c}
e_{1} \\
e_{2} \\
e_{3} \\
\vdots \\
\vdots \\
e_{k-1} \\
e_{k}
\end{array}\right]
$$

Equation (11) can be written as:

$$
Q=X A+E
$$

where $\mathrm{Q}$ is output vector, $\mathrm{X}$ is the input matrix, $\mathrm{A}$ is the weight vector and $\mathrm{E}$ is the combination error vector.

This equation can be treated as a multiple linear regression model. The ordinary least squares (OLS) estimate the weights vector $\hat{A}$ is given by:

$$
\hat{A}=\left[X^{T} X\right]^{-1} X^{T} Q
$$

In the WAM, the sum of weights a is normally constrained to unity (Shamseldin et al 1997, Dickinson 1975) i.e.

$$
\sum_{i=1}^{N} a_{i}=1
$$

The OLS solution of equation (13) may not ensure the satisfaction of the constraints of equation (14) thus the method of constrained least squares (CLS) can be used to estimate the weights vector (Shamseldin et al 1997, Bruen 1985) as:

$$
\hat{A}_{c l s}=\left[X^{T} X\right]^{-1}\left[X^{T} Q+\frac{1}{2} b \lambda\right]
$$


where $\mathrm{b}$ is a unit vector having same dimension as the vector $\mathrm{A}$ and $\lambda$ is the Lagrange multiplier given by (Shamseldin et al 1997):

$$
\lambda=2\left[b^{T}\left(X^{T} X\right)^{-1} b\right]^{-1}\left[1-b^{T}\left(\left(X^{T} X\right)^{-1}\right) X^{T} Q\right]
$$

Shamseldin et al (1997) and Winkler (1989) pointed out that the main disadvantage of the WAM is that it may suffer from multi-collinearity problem which results in unstable estimates of the weights reducing the advantages obtained from combining the different models forecasts. The degree of multi-collinearity increases with the increase in the forecasting ability of the individual models as well as when the forecasts of the individual models used are very similar not necessarily being good.

\subsubsection{The Neural Network Method (NNM)}

SAM and WAM methods provide a relatively simpler methods of combining the forecasts. An alternative method is the NNM which can be used to test whether a more complex relationship such as a nonlinear function mapping of inputs into the network output, is needed for the combinations (Shamseldin et al 1997).

There are several types of neural networks available, however, the type used in this study is multi layer feedforward network. This type is very powerful in function modelling (Shamseldin et al 1997).

The multi layer feedforward neural network used consists of an input layer, an output layer and only one hidden layer between the input and output layers. A layer is usually a group of neurons having same pattern of connection pathways to the other neurons of adjacent layers. Each neuron in a particular layer has connection pathways to all the neurons in the next adjacent layer but non to those of the same layer (figure (2)). 


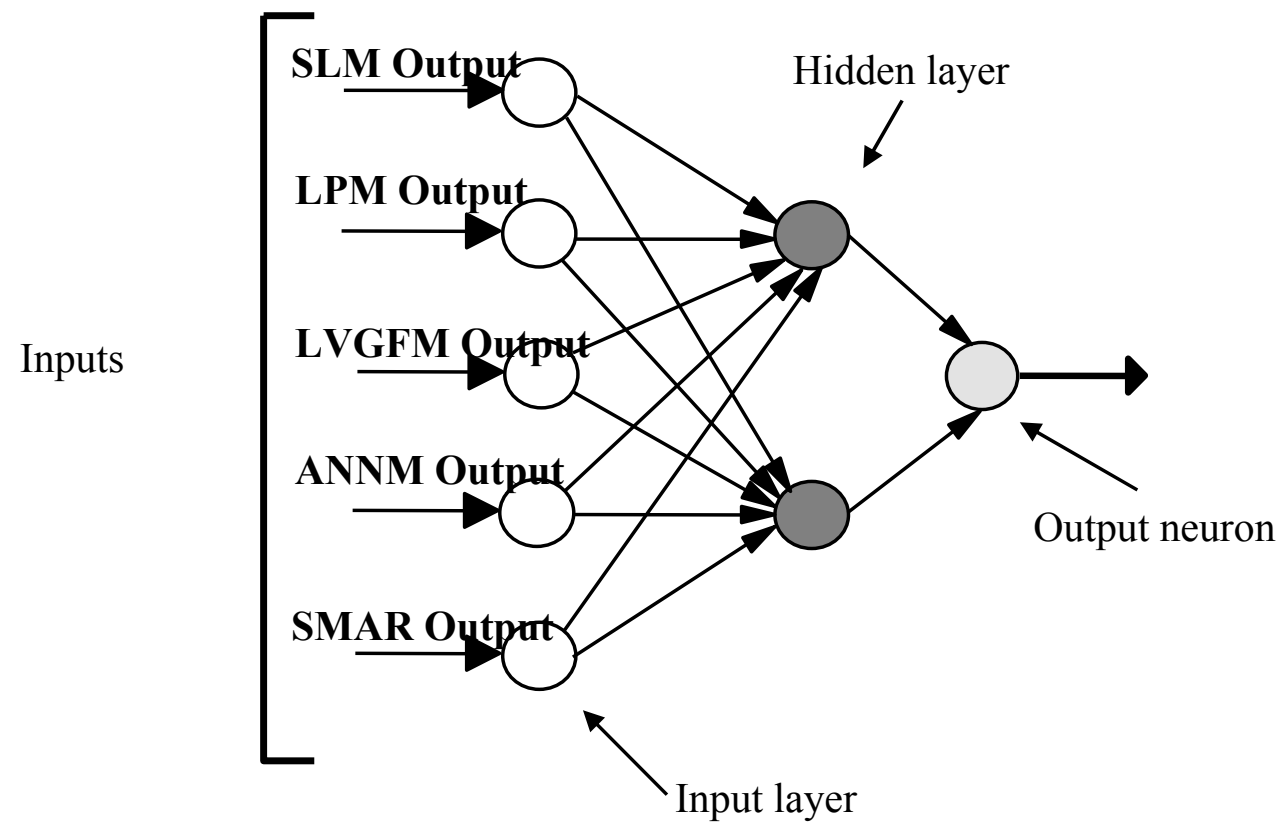

Figure (2): Schematic diagram of the Artificial Neural Network model

The number of neuron in the input layer is equal to the number of elements in the external input array to the network. In this study the elements of the external array are the forecast of selected models each of which is assigned to only one neuron. These inputs were transformed to outputs using a transfer function given by:

$$
f\left(X_{i}\right)=X_{i}
$$

where $X_{i}$ is the $i^{\text {th }}$ external input to the $i^{\text {th }}$ neuron in the input layer. The outputs of these neurons in the input layer are distributed through connection pathways to the neurons of the single hidden layer.

The hidden neurons has no direct connection with either the external input or output of the network. Each neuron in the hidden layer receives its input through connection pathways from the neurons of the input layer and transmit their output along the connection pathways to all the neurons of the output layer. The output layer in turn accumulates the transmitted input and 
produce the network output. The number of neurons in the output layer equals the number of outputs expected from the network.

A neuron in the hidden or output layer receives inputs and transform it to output $\mathrm{y}_{\mathrm{o}}$ by a mathematical transfer function given by equation (7).

These network parameters are usually estimated by a procedure referred to in neural networks as training (Shamseldin et al 1997 and Hammestrom 1993) analogous to the calibration procedure in hydrological modelling.

The transfer function is usually nonlinear and the most widely used one is the logistic function given by equation (8).

\section{The Model Efficiency Criteria}

In this study, the performances of the selected models (SLM, LPM, LVGFM, ANN and SMAR) as well as the three methods of combining model outputs (SAM, WAM and NNM) are all evaluated using $\mathrm{R}^{2}$ model efficiency criteria of Nash and Sutcliffe (1970). The $\mathrm{R}^{2}$ reflects the amount of initial variance accounted for by the model. So it is closely linked to the sum of squares of the differences $F$ between the estimated and observed discharges. The criterion is defined by:

$$
R^{2}=1-\frac{F}{F_{0}}=1-\frac{M S E}{M S E_{0}}
$$

where $\mathrm{F}_{0}$ is the initial variance of the discharges about their mean over the calibration period or some times called the initial mean square error $\left(\mathrm{MSE}_{0}\right)$ given by:

$$
F_{0}=M S E_{0}=\frac{1}{N} \sum_{i=1}^{N}\left(q_{i}-\bar{q}\right)^{2}
$$

where $\bar{q}$ is mean observed discharge over the calibration period.

$\mathrm{F}$ is the residual model variance (variance not accounted for by the model) or some times called the residual mean square error (MSE). It reflects the sum of the squares of the differences between the observed discharges $\mathrm{q}_{\mathrm{i}}$ and the model estimates $\hat{q}_{i}$ given by: 


$$
F=M S E=\frac{1}{N} \sum_{i=1}^{N}\left(q_{i}-\hat{q}_{i}\right)^{2}
$$

The initial variance $\mathrm{F}_{0}$ can be visualized as the variance of a naïve model having a forecast all the time equal to the mean of the observed discharge. The quantities $\mathrm{F}$ and $\mathrm{F}_{0}$ for the calibration period are estimated over that period. For the verification period the residual variance $F$ is calculated over the period itself while the initial variance $F_{0}$ is calculated using the mean discharge over the calibration period. The idea behind this calculation is that the naïve forecast in the verification period is the mean discharge of the calibration period.

Therefore, the $\mathrm{R}^{2}$ can be regarded as a measure of the performance of the substantive relative to that of the naïve (primitive) model.

\section{The Study Area}

Blue Nile catchment up to Eddeim was used in this study the details of which are shown in table 1 . The catchment represents the main source of the Blue Nile namely the Ethiopian high lands. The Blue Nile up to Eddeim has an area of $176,572 \mathrm{~km}^{2}$. Topographically, there is a wide variation which well represents the topography of the Nile basin. Climatologically, the selected catchment represents the whole scale from the arid to the tropical zones where the mean annual rainfall varies from few hundreds of millimetres to some thousands of millimetres.

Table 1: Information about the catchment used in the study and its concurrent data sets

\begin{tabular}{|c|c|c|c|c|c|c|c|}
\hline \multirow[t]{2}{*}{ Basin } & \multirow{2}{*}{$\begin{array}{c}\text { Area } \\
\left(\mathbf{k m}^{2}\right)\end{array}$} & \multirow[t]{2}{*}{ Country } & \multirow{2}{*}{$\begin{array}{c}\text { Rainfall } \\
\text { Stations } \\
\text { Used }\end{array}$} & \multicolumn{4}{|c|}{ The data sets } \\
\hline & & & & $\begin{array}{l}\text { No. } \\
\text { years }\end{array}$ & $\begin{array}{l}\text { Starting } \\
\text { year }\end{array}$ & Calibration & Verification \\
\hline $\begin{array}{l}\text { Blue } \\
\text { Nile }\end{array}$ & 176,572 & Ethiopia/Sudan & 9 & 7 & 1990 & $\begin{array}{c}\text { Jan 1990-Dec } \\
1994\end{array}$ & $\begin{array}{c}\text { Jan 1995-Dec } \\
1996\end{array}$ \\
\hline
\end{tabular}

Data of 10 daily recording rain gauging stations, that are located within the basin and just outside it, were used. The details, associated with each station, like station number; length of the available record etc. are presented in table 2. The source of the rainfall data is the Ministry of Irrigation and Water Resources of the Sudan and USGS FEWS NET. 
Table 2: Details of the 10 rainfall stations

\begin{tabular}{|l|r|r|r|r|r|}
\hline Station & Starting year & \multicolumn{1}{|c|}{ No. of years } & No. of data points & \% missing & Mean annual rainfall \\
\hline GONDAR & 1987 & 12 & 4383 & 24 & 1020 \\
\hline MAKALE & 1987 & 12 & 4383 & 44 & 625 \\
\hline BAHAR DAR & 1987 & 12 & 4383 & 19 & 1230 \\
\hline COMBOL-CHA & 1987 & 12 & 4383 & 22 & 1020 \\
\hline DEBRE MARKOS & 1987 & 12 & 4383 & 24 & 1310 \\
\hline ADDIIS ABABA & 1987 & 12 & 4383 & 14 & 1180 \\
\hline LEKEMETI & 1987 & 12 & 4383 & 49 & 2140 \\
\hline GORE & 1987 & 12 & 4383 & 47 & 1746 \\
\hline JIMMA & 1987 & 12 & 4383 & 36 & 1607 \\
\hline HARRAR MEDA & 1987 & 12 & 4383 & 41 & 860 \\
\hline
\end{tabular}

Mean Annual Rainfall (MAR) defined as the mean rainfall that a basin receives in a year. Currently, it was estimated using the methods of Arithmetic mean.

The method of Arithmetic Mean calculates the sum of all daily observations divided by the total number of observations. This figure multiplied by 365 is the MAR. Observations are summed for all stations with records within or just outside the basin. The MAR for the basin as calculated by arithmetic mean was found to be $1245 \mathrm{~mm}$.

The method of Simple Arithmetic Mean was used to estimate time series of Average Daily Rainfall (ADAR) over the catchment. The method calculates ADAR as sum of all rainfall observations made on day d divided by the number of observations made on the day. For ten rain gauges it is not always necessary that on each day all 10 observations were made. If only 8 observations were made on day $\mathrm{d}$, then the sum of observations made on that day was divided by 8 to give ADAR for that day.

Limited amounts of climatic data are available for estimation of potential evaporation in the basin. Data are available on minimum and maximum daily temperature, wind speed, sunshine hours and relative humidity. Data on all parameters that are required in Penman calculations are available for only five years of early nineties. The mean annual evaporation over the catchment was found to be $1400 \mathrm{~mm}$

The flow data is collected from the Ministry of Irrigation and water resources of the Sudan. The available data goes back to 1964 . 
Unfortunately, after 1996 the area becomes inaccessible due to security reasons and hence no data available beyond the end of 1996.

The total recorded data available is for 33 years with only $2 \%$ missing. The missing part is mainly in the year 1988 (very wet year). It has along term mean annual value of $195 \mathrm{~mm}$ (about 570000 cumecs)

Figure (3) shows the Blue Nile up to Eddeim catchment and river network along with the locations of rainfall gauges and flow gauging station at Eddeim.

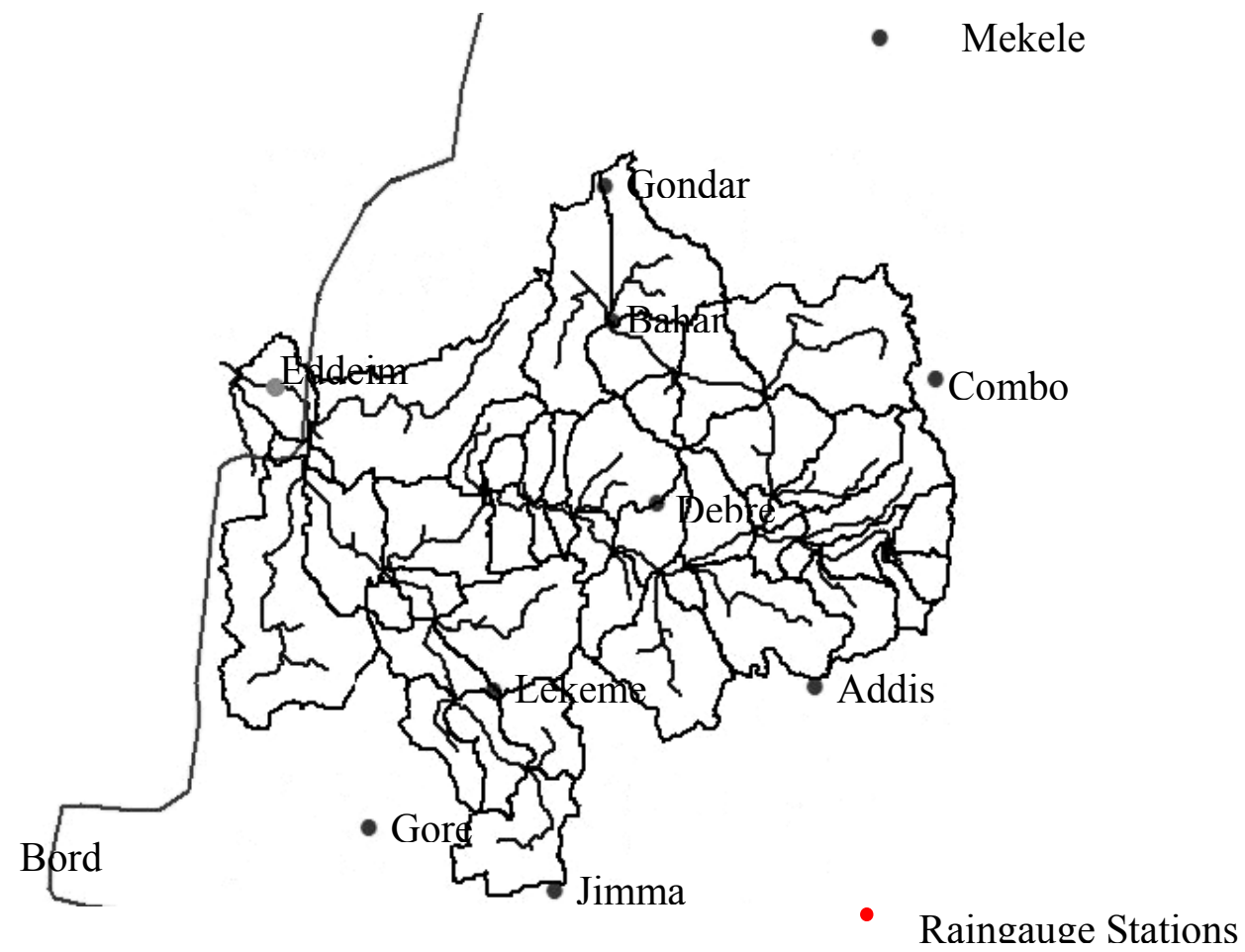

Figure (3): Blue Nile at Ed-deim catchment, rain gauge and the River Network

The catchment hydrologic diagram presents the expected variation through the year of rainfall, potential evaporation and discharge. Figure (4) gives an idea about the water balance of the basin. From the figure one can 
see that the effective rainy season (rainfall exceeds potential evaporation) can be considered from May to September. It can also be seen that the peak of the rainfall occurs on average three weeks before the peak of the flow. The flow hydrograph starts to pick up in two weeks time after the start of the effective rainy season.

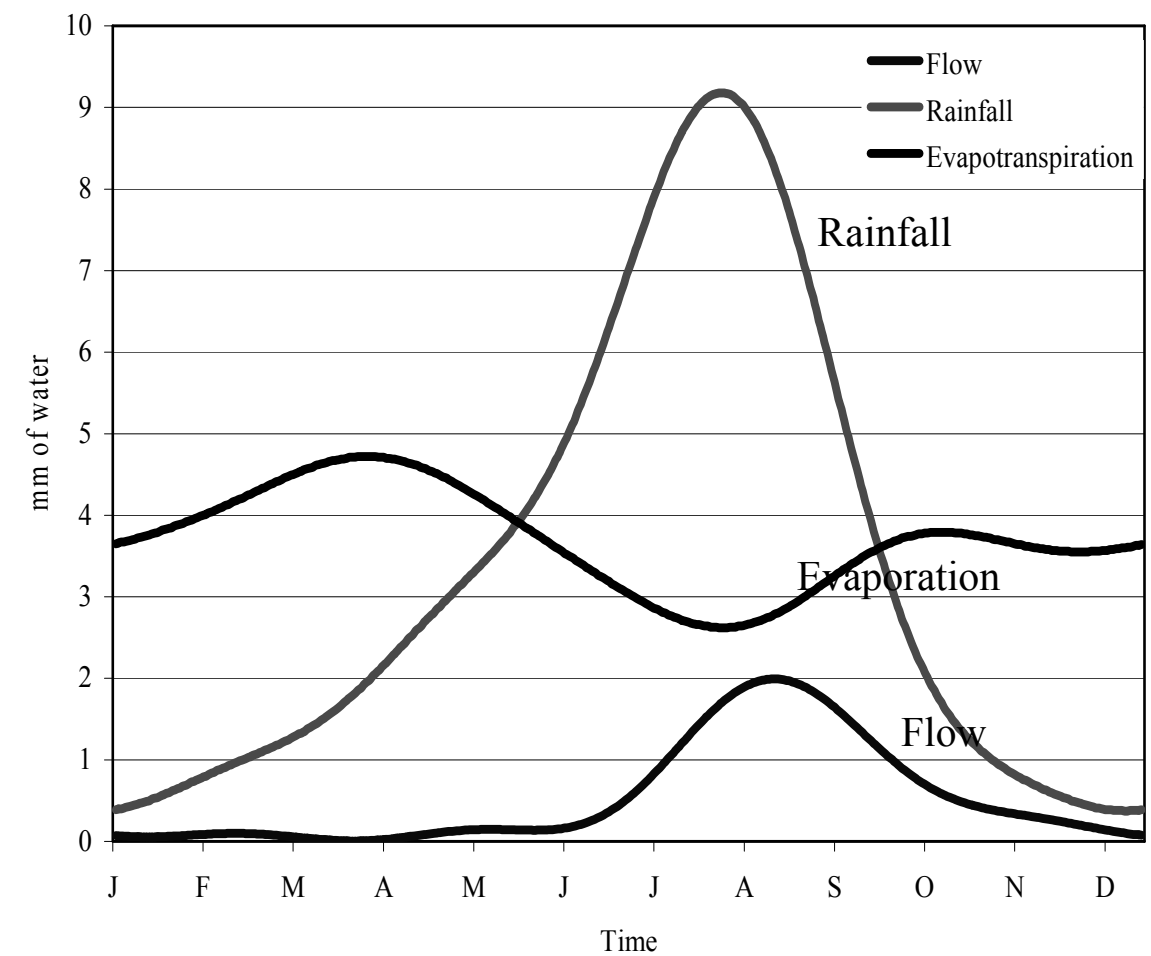

Figure (4): Hydrologic Diagram of Blue Nile Ed-Deim Catchment

\section{Application, Results and Discussion}

Each of the five basic models is applied to each of the selected catchment, using split-record evaluation, involving the use of calibration and verification periods. Roughly about two-thirds of the available data is used for calibration and one-third for verification. The method of combining outputs is also applied to the selected catchment using the outputs of the basic models applied in simulation mode (as distinct from the updating mode). In terms of increasing complexity, the SLM is the simplest, followed 
by the LPM in their non-parametric and parametric forms and the LVGFM. These three are system-theoretic in structure, and ordinary least squares solution is used for estimation of the pulse response function except for the parametric forms where the parameters were optimised. The SMAR model parameters are estimated using the three optimisation techniques, simplex, Rosenbrock and genetic algorithm. As for the ANN, the number of weights depends on the number of neurons chosen in the input layer and the hidden layer. If ' $l$ ' is the total number of neurons in the input layer and ' $m$ ' is that in the hidden layer, then the total number of weights to be estimated is $(l+1) \mathrm{m}$ $+(m+1)$ (Shamseldin et al 1997). As such, the ANN is the least parsimonious amongst the models chosen in the study. The simplex method of automatic optimisation is also used for the calibration of the ANN. The results of performances of the substantive models are shown in Table 3 and 4 respectively for the simulation and updating modes.

From these results, it is clear that the simulation mode performance of the naïve SLM is inferior to that of all other models. As expected, the LVGFM, which is a modification of the SLM, incorporating an element of linear variation of the gain factor $G_{t}$ with the catchment wetness index at each time-step, performs better than the SLM. In the case of very large catchments, such as Blue Nile, the LVGFM, SMAR and ANN models have same order of performance with a slightly less efficiency in the case of SMAR. This is due to the lumped-parameter effect in the SMAR model applied to a large catchment. The over-parameterisation in the case of ANN affects its performance.

For catchments characterised by physiographical and hydrometeorological variability; and displaying strong seasonality, the LPM in simulation mode, with its inherent component of seasonal variation, outperforms the SMAR, LVGFM and the ANN models.

The SLM and LPM can also be applied in the updating mode in addition to the MOCT. The method of combined outputs is applied to the results of the simulation modes of the five substantive models. Table 4 shows the performance results of the models in updating mode. It can be seen that the LPM consistently performed better than all the other models. It accounted for more than $90 \%$ of the initial variance.

These results indicate that simple models, involving fewer parameters or weights to be evaluated, and relying on simple mathematical procedures (e.g. the ordinary least squares solution), are often better in discharge forecasting than models which involve a significantly higher 
number of parameters or weights to be evaluated and which rely on complex mathematical computations (e.g. automatic optimization).

Table (3): Results of model performance in simulation mode

\begin{tabular}{|l|l|l|l|l|l|l|}
\hline \multirow{2}{*}{ Period } & Basin & \multicolumn{5}{|c|}{ Substantive Model } \\
\cline { 3 - 7 } & & SLM & LPM & VGFM & SMAR & ANN \\
\hline Calibration & Blue Nile & 77.8 & 92.1 & 91.2 & 90.5 & 91.8 \\
\hline Verification & Blue Nile & 76.0 & 91.1 & 89.0 & 89.2 & 90.7 \\
\hline
\end{tabular}

Table (4): Results of model performance in updating mode

\begin{tabular}{|l|l|l|l|l|l|l|l|l|l|}
\hline Period & Basin & Method & SLM & LPM & VGFM & \multicolumn{2}{|l|}{ SMAR } & \multicolumn{3}{|c|}{ MOCT } \\
\hline Calibration & $\begin{array}{l}\text { Blue } \\
\text { Nile }\end{array}$ & Parametric & 98.2 & 98.6 & 89.8 & 92.4 & 89.9 & 92.4 & 92.4 \\
\hline Verification & $\begin{array}{l}\text { Blue } \\
\text { Nile }\end{array}$ & Parametric & 97.3 & 97.2 & 88.4 & 91.6 & 88.4 & 91.6 & 91.5 \\
\hline
\end{tabular}

\section{Conclusions}

The performance of the naïve SLM is clearly inferior to that of all other models. For the Blue Nile catchment which is characterized by strong seasonality, the LPM outperforms the LVGFM. For large catchments with such seasonality, the LPM performance is even better than the SMAR model. The ANN, although characterized by a large number of weights (parameters), does not generally perform better than the simpler models. The SMAR model variants, having either nine or ten parameters, fail to adequately simulate the hydrological behaviour of the large catchment of the Blue Nile.

In conclusion, this study confirms that simpler models for continuous river-flow simulation can surpass their complex counterparts in performance. There is a strong justification; therefore, for the claim that increasing the model complexity, thereby increasing the number of parameters, does not necessarily enhance the model performance. Therefore, in practical hydrology, the simple models, can still play a significant role as effective simulation tools, and that performance enhancement is not guaranteed by the adoption of complex model structures. 
As a concluding statement, LPM in its updating mode can be used to safely forecast flows in the Blue Nile River with a performance efficiency of more than $90 \%$ i.e. accounting for more than $90 \%$ of the initial variance.

\section{References}

1. Ahsan, M. and K.M. O'Connor, "A simple nonlinear rainfall-runoff model with a variable gain factor", Journal of Hydrology, 155: 151-183, 1994.

2. Armstrong, J. S., "Combining forecasts: the end of the beginning or the beginning of the end?", Int. J. Forecasting, 5: 585-588, 1989.

3.Bruen, M., "Black box methods of systems applied to modelling of catchment behaviour." Ph.D. thesis, National University of Ireland, 1985.

4.Clarke, R.T., "Statistical Modelling in Hydrology", John Wiley and Sons, 1994.

5.Granger, C. W. J. and Ramanathan, R., "Improved methods for combining forecasts." J. Forecasting 3: 197-204, 1984.

6.Hammestorm, D., "Working with neural networks." IEEE spectrum, 30: 46-53, 1993.

7.Kachroo, R.K. and G.C. Liang, "River flow forecasting, Part 2, Algebraic development of 480 linear modelling techniques," Journal of Hydrology, 133: 17-40, 1992.

8.Makridakis, S. and Winkler, R., "Average of Forecasts: some empirical results, Manage." Sci., 29(9): 987-996, 1983.

9.Makridakis, S., Andersen, A., Carbone, R., Fildes, R. Hibon, M., Lewandowski, R., Newton, J., Parzen, E. and Winkler, R., "The accuracy of extrapolation (time series) methods": results of a forecasting competition. J. Forecasting, 1: 111-153, 1982.

10.Nash, J.E. and J.V. Sutcliffe, "River flow forecasting through conceptual models, Part1, A discussion of principles," Journal of Hydrology, 10: 282290, 1970.

11.Nash, J.E. and J.J. Foley, "Linear models of rainfall-runoff systems. In: 'Rainfall-Runoff Relationship", Proceedings of the International Symposium on Rainfall-Runoff modelling. Mississippi State University, May 1981, USA. Edited by V.P. Singh, Water Resources Publications pp.: 51-66, 1982.

12. Nash, J.E. and B.I. Barsi, "A hybrid model for flow forecasting on large catchments", Journal of Hydrology, 65: 125-137, 1983. 
13. O'Connell, P.E., J.E. Nash, and J.P. Farrell, "River Flow forecasting through conceptual models." Part 2. The Brosna catchment at Ferbane, Journal of Hydrology., 10: 317-329,

1970.

14. O’Connor, K.M., M. Goswami, G.C. Liang, R.K. Kachroo, and A.Y. Shamseldin, "The Development of The "Galway Real-Time River Flow Forecasting System (GFFS)," The proceedings of the 19th European Regional Conference of ICID, Czech Republic, 2001.

15. Shamseldin, A. Y., "Application of a neural network technique to rainfall-runoff modelling", Journal of Hydrology, 199, 272-294, 1997.

16.Shamseldin, A.Y., K.M. O'Connor, and G.C. Liang, "Methods for combining the outputs of different rainfall-runoff models." Journal of Hydrology, 197: 203-229, 1997.

17.Winkler, R. L., Combining forecasts: "A philosophical basis and some current issues." Int. J. Forecasting, 5: 605-609, 1989. 